\title{
Comparação entre os Achados Ultra-sonográficos, Histeroscópicos e Histopatológicos no Sangramento Uterino da Pós-menopausa
}

Comparison of Ultrasonographic, Hysteroscopic and Histopathologic Findings in Women with Postmenopausal Uterine Bleeding

Adriana Scavuzzi ${ }^{1}$, Melania Amorim ${ }^{1}$, João Sabino Pinho Neto ${ }^{2}$, Luis Carlos Santos ${ }^{1}$

\section{RESUMO}

Objetivos: determinar a associação entre os achados ultra-sonográficos, histeroscópicos e histopatológicos em mulheres com sangramento uterino na pós-menopausa.

Métodos: realizou-se estudo descritivo, retrospectivo, tipo transversal, incluindo 156 pacientes com sangramento uterino na pós-menopausa atendidas no Centro Diagnóstico do Instituto Materno-Infantil de Pernambuco no periodo de janeiro de 1995 a dezembro de 2001. Os resultados ultra-sonográficos foram classificados como anormais ou normais, de acordo com os pontos de corte de 4 e $5 \mathrm{~mm}$. Determinou-se a freqüência dos principais achados histeroscópicos e histopatológicos destas mulheres, categorizados como lesões pré-malignas/ malignas (hiperplasias/câncer) e lesões benignas. Determinou-se ainda a concordância entre os achados histeroscópicos e histopatológicos.

Resultados: a freqüencia de espessamento do eco endometrial de acordo com os pontos de corte de $4 \mathrm{~mm}$ e $5 \mathrm{~mm}$ foi de $75 \%$ e de 67,3\%, respectivamente. O achado histeroscópico mais freqüente foi o endométrio atrófico (37,8\%), seguindo-se os pólipos endometriais $(35,9 \%)$, hiperplasia do endométrio (10,9\%), câncer de endométrio (10,3\%), endométrio funcionante (30.2\%) e outros achados (1,9\%). Em relação aos achados histopatológicos, observou-se também maior freqüência do endométrio atrófico (31,4\%), seguindo-se os pólipos endometriais (26,3\%), material insuficiente (16,0\%), câncer de endométrio (10,9\%), hiperplasia $(9,0 \%) e$ outros achados (6,4\%). Observou-se associação estatisticamente significante entre espessamento endometrial e presença de lesões pré-malignas e malignas. Observou-se um único caso de malignidade em pacientes com ponto de corte de $5 \mathrm{~mm}$, e nenhum caso foi evidenciado com o ponto de corte de $4 \mathrm{~mm}$. Encontrou-se uma boa taxa de concordância entre os achados histeroscópicos e histopatológicos (kappa = 0,61).

Conclusões: a freqüência de eco endometrial espessado foi de $75 \%$ e de 67,3\% com os pontos de corte de $4 \mathrm{~mm}$ e $5 \mathrm{~mm}$, respectivamente. Nenhum caso de lesão pré-maligna ou maligna foi observado quando foi adotado o ponto de corte de $4 \mathrm{~mm}$. Os achados histeroscópicos e histopatológicos mais freqüentes foram o endométrio atrófico e os pólipos endometriais, observando-se boa taxa de concordância entre esses exames.

PALAVRAS-CHAVE: Sangramento na pós-menopausa. Espessura endometrial. Ultrasonografia. Histeroscopia. Endométrio: avaliação.

\footnotetext{
${ }^{1}$ Instituto Materno-Infantil de Pernambuco (IMIP)

${ }^{2}$ Universidade Federal de Pernambuco (UFPE)

Correspondência:

Melania Maria Ramos de Amorim

Rua Neuza Borborema de Sousa, 300 - Bairro Santo Antônio

58103-313 - Campina Grande - Paraíba

e-mail: melamorim@uol.com.br
}

\section{Introdução}

A menopausa é definida como a última menstruação ovário-dependente da vida de uma mulher e representa apenas um ponto, embora marcante, dentro de um estado bem mais amplo chamado climatério. Este, por sua vez, marca a 
transição do período reprodutivo para o não reprodutivo $^{1,2}$.

O sangramento na pós-menopausa é definido quando ocorre doze meses após a última menstruação e é responsável por cerca de 5\% das consultas ginecológicas no climatério. Além disto, é o principal sintoma do câncer de endométrio ${ }^{3}$, sendo em $80 \%$ dos casos o primeiro sinal desta neoplasia ${ }^{4}$.

Nos Estados Unidos da América, o câncer de endométrio é a quarta neoplasia mais freqüente entre as mulheres, com uma estimativa de 34.000 novos casos diagnosticados e 6.000 óbitos no ano de $1996^{3}$. No Brasil, são esperados anualmente 5.685 casos novos de adenocarcinoma de endométrio, com taxa de 7,6 casos por 100.000 mulheres, variando de 2,0/100.000 na Região Norte a 9,9/ 100.000 na Região Sudeste. Espera-se aumento da incidência nas próximas décadas, decorrente de aumento da longevidade associado a freqüência elevada de alguns fatores de risco como a obesidade $^{5,6}$.

Apesar da considerável possibilidade de uma paciente com sangramento uterino na pós-menopausa apresentar doença benigna, segundo $\mathrm{Hamou}^{7}$, a investigação endometrial é obrigatória, uma vez que todo processo maligno e pré-maligno deve ser excluído. Com este objetivo, varias técnicas vêm sendo utilizadas para avaliar a mucosa uterina.

Apesar de ainda ser considerada em diversos estudos como padrão-ouro para investigação do sangramento na pós-menopausa ${ }^{8}$, a curetagem uterina representa procedimento invasivo, necessita de hospitalização, uso de anestesia e possui custo elevado. Por ser exame realizado às cegas (sem visão direta da cavidade uterina), é comumente associado a falhas diagnósticas, com taxa de falso-negativo que varia de 2 a $10 \%{ }^{8,9}$. Stock e Kanbur $^{10}$ evidenciaram que em $60 \%$ das curetagens realizadas menos da metade da cavidade uterina era curetada e em 16\% dos casos menos de um quarto do endométrio havia sido estudado, questionando a acurácia deste método ${ }^{10}$.

A histeroscopia permite a visualização direta de anormalidades difusas ou focais da cavidade endometrial. É procedimento bem tolerado, realizado ambulatorialmente e com boa acurácia na investigação do sangramento uterino anor$\mathrm{mal}^{9,11}$. No entanto, a histeroscopia tem o inconveniente de ser procedimento invasivo, além de possuir taxa de falso-negativos em torno de $3 \%{ }^{9}$.

Outros métodos têm sido propostos com a finalidade de avaliar a cavidade uterina de mulheres com sangramento uterino anormal. A ultrasonografia representa a primeira escolha entre os métodos não invasivos que permitem avalia- ção do endométrio. Vários estudos mostram a acurácia deste exame em afastar processos endometriais pré-malignos e malignos ${ }^{9,11,12}$. A acurácia do exame relaciona-se com o fato de que o câncer de endométrio geralmente associa-se a espessamento endometrial e raramente encontra-se presente em endométrios finos ${ }^{12,13}$.

Vários estudos têm sido publicados com o intuito de avaliar a espessura endometrial que seguramente afastaria um adenocarcinoma de endométrio. Estes estudos mostram que medida em torno de $4 \mathrm{~mm}$ ou $5 \mathrm{~mm}$ possui sensibilidade em torno de $95 \%$ para a detecção desta neoplasia uterina $^{12,13}$. Desta forma, espessura endometrial menor ou igual a $4 \mathrm{~mm}$ ou $5 \mathrm{~mm}$ representaria diagnóstico confiável de atrofia endometrial, afastando com segurança a possibilidade de doença endometrial. Nos casos em que a espessura ultrapassa o ponto de corte, a recomendação é de que se realizem outros métodos complementares, preferentemente a histeroscopia com biópsia dirigida, para se estabelecer o diagnóstico definitivo ${ }^{9}$.

Consideramos importante que os diversos serviços avaliem a acurácia dos métodos diagnósticos utilizados, uma vez que podem ocorrer variações tanto na técnica como nos equipamentos e na formação dos profissionais envolvidos. No presente estudo, realizado no Instituto Materno-Infantil de Pernambuco, em Recife, pretendemos determinar a associação entre os achados histeroscópicos e histopatológicos em pacientes com sangramento uterino na pós-menopausa, identificando a espessura do eco endometrial que justificaria avaliação uterina invasiva nestas pacientes.

\section{Pacientes e Métodos}

Realizou-se estudo descritivo, retrospectivo, tipo transversal, analisando os prontuários das pacientes com sangramento uterino espontâneo na pós-menopausa que foram submetidas a ultra-sonografia com medida da espessura do eco endometrial, seguida de histeroscopia diagnóstica com biópsia dirigida do endométrio, no Centro Diagnóstico do Centro de Atenção Mulher (CAM) do Instituto Materno-Infantil de Pernambuco (IMIP), em Recife.

Foram incluídas 156 pacientes no período de janeiro de 1995 a dezembro de 2001. Os critérios de inclusão foram: idade maior que 40 anos, pelo menos doze meses de amenorréia e história de sangramento uterino no período máximo de seis meses antes do inicio das investigações endometriais, sem uso de terapia de reposição hormonal.

As variáveis de análise foram os resultados da medida do eco endometrial pela ultra-sonogra- 
fia transvaginal, considerando-se dois pontos de corte: $4 \mathrm{~mm}$ e $5 \mathrm{~mm}$; os achados histeroscópicos e os resultados histopatológicos, incluindo tanto as lesões pré-malignas (hiperplasia de endométrio) e malignas (câncer de endométrio) como achados benignos (miomas submucosos, pólipos, endométrio atrófico, endométrio funcionante e outros). Classificaram-se os achados ultra-sonográficos como normais ou anormais, de acordo com a espessura medida do endométrio, conforme os pontos de corte já mencionados.

Todos os exames ultra-sonográficos foram realizados ambulatorialmente no IMIP utilizandose equipamento do tipo Aloka SSD-500 com transdutor transvaginal com emissão de freqüência de $5 \mathrm{MHz}$ e ângulo de abertura de $90^{\circ}$ (Aloka Co., Toquio, Japão). A medida do eco endometrial foi feita na sua porção mais larga num plano longitudinal e incluía ambas as camadas do endométrio. O exame foi sempre realizado por um dos especialistas em ultra-sonografia do Centro Diagnóstico, CAM-IMIP.

A histeroscopia era realizada rotineiramente após a ultra-sonografia e o intervalo entre estes dois exames foi sempre inferior a três meses. Foram utilizados histeroscópios rígidos com ótica de $30^{\circ}$, com camisa diagnóstica de $5 \mathrm{~mm}$ (Richard Wolf Medical Instrument Corp., Rosemont, IL) para avaliação da cavidade uterina e escolha das áreas a serem biopsiadas. Todos os procedimentos foram realizados no Centro Diagnóstico do CAM-IMIP por um dos especialistas em endoscopia ginecológica, utilizando-se como meio distensor o dióxido de carbono ou soro fisiológico, tendo sido empregadas sedação ou raquianestesia, conforme critério clínico.

O material endometrial obtido por biópsia durante a histeroscopia foi encaminhado ao Departamento de Anatomia Patológica do IMIP e avaliado histologicamente, tendo sido os blocos de parafina cortados em micrótomos ajustados a $3 \mu \mathrm{m}$ de espessura, sendo as lâminas submetidas a coloração por hematoxilina-eosina. Avaliou-se, do ponto de vista morfológico, toda a extensão do corte histológico.

Os dados foram analisados utilizando-se o software de domínio público Epi-Info 2000. Utilizou-se o teste $\chi^{2}$ para determinar a associação entre os achados ultra-sonográficos de medida do eco endometrial (pontos de corte de $4 \mathrm{~mm}$ e $5 \mathrm{~mm}$ ) com os resultados histológicos (lesões pré-malignas/malignas ou achados benignos), adotando-se o nível de significância de 5\%. Para avaliação da concordância foi utilizado o teste de kappa. Os valores deste variam de 0 a 1 , sendo que 0 indica concordância não melhor do que a encontrada pelo acaso e 1, concordância perfeita.
A presente pesquisa foi realizada obedecendo às normas e diretrizes éticas da Resolução 196/ 96 do Conselho Nacional de Saúde do Ministério da Saúde, em que é garantido o caráter confidencial da identidade das pacientes e das informações obtidas neste estudo. O projeto original foi aprovado pelo Comitê de Ética em Pesquisa do IMIP.

\section{Resultados}

As medidas do eco endometrial foram agrupadas e classificadas em achados normais e anormais, de acordo com os pontos de corte de $4 \mathrm{~mm}$ e $5 \mathrm{~mm}$. Quando estabelecido o ponto de corte de 4 $\mathrm{mm}$ para a espessura do eco endometrial, 75\% das mulheres tiveram as medidas da espessura do endométrio consideradas anormais, diminuindo para $67,3 \%$, com o ponto de corte de $5 \mathrm{~mm}$.

Considerando-se os resultados da histeroscopia em mulheres com sangramento uterino na pós-menopausa, esta foi classificada em anormal (21\%) e normal $(78,8 \%)$. O endométrio atrófico e o pólipo endometrial constituíram quase $75 \%$ dos achados histeroscópicos nestas pacientes, encontrando-se 21,2\% de lesões pré-malignas (hiperplasia) e malignas (câncer de endométrio) (Tabela 1).

Tabela 1 - Distribuição dos resultados da histeroscopia em mulheres com sangramento uterino na pós-menopausa.

\begin{tabular}{lrr}
\hline Histeroscopia & $\mathbf{n}$ & $\%$ \\
\hline Endométrio atrófico & 59 & 37,8 \\
Endométrio funcionante & 5 & 3,2 \\
Pólipo endometrial & 56 & 35,9 \\
Hiperplasia do endométrio & 17 & 10,9 \\
Câncer do endométrio & 16 & 10,3 \\
Outros & 3 & 1,9 \\
Total & 156 & 100 \\
\hline
\end{tabular}

Em relação ao exame histopatológico, encontrou-se uma freqüência de 19,9\% de resultados anormais, predominando entre os achados o endométrio atrófico $(31,4 \%)$. Observou-se ainda freqüência de pólipos em torno de $26 \%$, câncer de endométrio aproximadamente $11 \%$ e hiperplasia 9,0\% (Tabela 2).

Observou-se boa concordância entre os achados histeroscópicos e histopatológicos (kappa = 0,61) (Tabela 3).

Comparou-se a espessura do eco endometrial, com ponto de corte de $4 \mathrm{~mm}$, com os resultados do estudo histopatológico do endométrio. Es- 
tes, por sua vez, foram agrupados em lesões prémalignas e malignas. Todas as pacientes (100\%) com estudo do endométrio anormal (lesões prémalignas e malignas) encontravam-se na faixa de eco endometrial maior que $4 \mathrm{~mm}$. No entanto, 86 mulheres $(73,5 \%)$ com resultados do estudo histopatológico normal também estavam acima do ponto de corte. Encontrou-se diferença estatisticamente significativa entre os dois grupos para o ponto de corte de $4 \mathrm{~mm}$. Verificou-se, portanto, que nenhuma lesão endometrial pré-maligna ou maligna foi encontrada quando um ponto de corte de 4 $\mathrm{mm}$ para espessura do endométrio foi estabelecido como normal (Tabela 4).
Tabela 2 - Distribuição dos resultados do estudo histopatológico do endométrio em mulheres com sangramento uterino na pós-menopausa.

\begin{tabular}{lcc}
\hline Estudo histopatológico & $\mathbf{n}$ & $\%$ \\
\hline Endométrio atrófico & 49 & 31,4 \\
Material insuficiente, coágulos e fibrina & 25 & 16,0 \\
Pólipo endometrial & 41 & 26,3 \\
Câncer do endométrio & 17 & 10,9 \\
Hiperplasia do endométrio & 14 & 9,0 \\
Outros & 10 & 6,4 \\
Total & 156 & 100 \\
\hline
\end{tabular}

Tabela 3 - Correlação entre os achados histeroscópicos e histopatológicos nas pacientes com sangramento uterino anormal na pós-menopausa.

\begin{tabular}{|c|c|c|c|c|c|c|}
\hline Histeroscopia & Material escasso & Carcinoma & Hiperplasia & Pólipo & Outros & $\begin{array}{c}\text { Total } \\
59 \\
\end{array}$ \\
\hline Atrófico & 53 & 0 & 1 & 1 & & 16 \\
\hline Carcinoma & 1 & 13 & 2 & 0 & & 17 \\
\hline Hiperplasia & 3 & 3 & 7 & 3 & & 56 \\
\hline Pólipo & 13 & 0 & 4 & 37 & & 8 \\
\hline Outros & 4 & 1 & 0 & 0 & & \\
\hline Total & 74 & 17 & 14 & 41 & 10 & \\
\hline
\end{tabular}

kappa $=0,61$

Tabela 4 - Distribuição dos resultados da medida da espessura do eco endometrial, de acordo com o resultado do estudo histopatológico do endométrio (lesões pré-malignas e malignas), em mulheres com sangramento uterino na pós-menopausa, considerandose 0 ponto de corte de $4 \mathrm{~mm}$.

\begin{tabular}{|c|c|c|c|}
\hline \multirow{2}{*}{$\begin{array}{l}\text { Espessura do eco } \\
\text { endometrial }\end{array}$} & \multicolumn{3}{|c|}{ Lesões pré-malignas/malignas } \\
\hline & Sim & Não & Total \\
\hline Anormal (> 4 mm) & $31(26,5 \%)$ & $86(73,5 \%)$ & $117(100 \%)$ \\
\hline Normal $(<4 \mathrm{~mm})$ & $-(0 \%)$ & $39(100 \%)$ & $39(100 \%)$ \\
\hline Total & $31(19,9 \%)$ & $125(80,1 \%)$ & $156(100 \%)$ \\
\hline
\end{tabular}

Analisando-se o ponto de corte de $5 \mathrm{~mm}$, de acordo com os resultados do estudo histopatológico do endométrio (lesões pré-malignas e malignas), apenas uma paciente (2\%) com estudo do endométrio anormal apresentava eco endometrial abaixo de $5 \mathrm{~mm}$. No entanto, 75 (71,4\%) mulheres com resultado do estudo do endométrio normal também estavam acima do ponto de corte (Tabela 5).

\section{Discussão}

Os resultados deste estudo sugerem que a avaliação ultra-sonográfica da espessura endometrial é método bastante útil na triagem das pa-
Tabela 5 - Distribuição dos resultados da medida do eco endometrial, de acordo com o resultado do estudo do endométrio (lesões pré-malignas e malignas) em mulheres com sangramento uterino anormal na pós-menopausa, considerando-se o ponto de corte de $5 \mathrm{~mm}$.

\begin{tabular}{lcrr}
\hline $\begin{array}{l}\text { Espessura do } \\
\text { eco endometrial }\end{array}$ & \multicolumn{3}{c}{ Lesões } \\
Sim & \multicolumn{1}{c}{ Não } & \multicolumn{1}{c}{ Total } \\
\hline Anormal $(>5 \mathrm{~mm})$ & $30(28,6 \%)$ & $75(71,4 \%)$ & $105(100 \%)$ \\
Normal $(<5 \mathrm{~mm})$ & $1(2,0 \%)$ & $50(98,0 \%)$ & $51(100 \%)$ \\
Total & $31(19,9 \%)$ & $125(80,1 \%)$ & $156(100 \%)$ \\
\hline$\chi^{2}=15,3 \quad \mathrm{p}=0,0001$ & & &
\end{tabular}

cientes com sangramento uterino na pós-menopausa, identificando aquelas que devem ser submetidas a uma avaliação uterina invasiva. A atrofia $(31,4 \%)$ e os pólipos endometriais $(26,3 \%)$ foram os achados mais freqüentes nestas pacientes, ao passo que a freqüência de lesões pré-malignas e malignas foi de 9,0 e 10,9\%, respectivamente. Observou-se boa concordância entre os achados histeroscópicos e histopatológicos nas pacientes com sangramento uterino anormal.

É importante ressaltar que, embora a grande maioria dos casos de sangramento uterino na pós-menopausa sejam decorrentes de processos benignos ${ }^{14,15}$, todo sangramento nessa faixa etária deve ser investigado, uma vez que 5 a $15 \%$ dos casos devem-se à presença de câncer de endo- 
métrio $^{3,6}$. Os nossos resultados pertinentes à freqüência e à etiologia das lesões endometriais mais freqüentemente envolvidas na gênese do sangramento uterino anormal são concordantes com a literatura. De acordo com vários trabalhos publicados, o delgado e frágil endométrio encontrado nos casos de atrofia seria a principal causa de sangramento em pacientes na pós-menopau$\mathrm{sa}^{16,17}$. O sangramento nesses casos é facilmente justificável pelas características histológicas do endométrio, ocorrendo pela ruptura dos capilares arteriais e venosos protegidos pela fina camada endometrial.

Além da atrofia endometrial, uma gama de achados histológicos pode se associar ao sangramento uterino na pós-menopausa, abrangendo desde o endométrio proliferativo e lesões benignas (pólipos, miomas) até as lesões pré-malignas e malignas. No maior estudo multicêntrico prospectivo avaliando sangramento uterino na pósmenopausa, Karlsson et al. ${ }^{13}$ incluíram 1.168 mulheres e encontraram freqüência de atrofia endometrial de 59\%, enquanto as outras causas foram representadas por pólipos (12,4\%), câncer de endométrio $(10,1 \%)$, hiperplasias $(9,9 \%)$ e endométrio funcionante $(6,8 \%)$.

Encontramos em nossa pesquisa freqüência de pólipos endometriais bem maior do que a relatada por Karlsson et al. ${ }^{13}$. Esta diferença encontrada provavelmente deve-se ao fato de que tanto nesse como em outros grandes estudos a cavidade uterina foi avaliada por meio de curetagem uterina, ao passo que em nosso estudo se adotou a histeroscopia com biópsia dirigida. A curetagem, realizada às cegas, representa método com baixa acurácia em relação à histeroscopia, para o diagnóstico de lesões endometriais focais ${ }^{18,19}$. A freqüência de lesões pré-malignas e malignas em nosso estudo foi concordante com a encontrada na literatura ${ }^{13,18}$. Cumpre destacar que encontramos uma freqüência muito semelhante de câncer $(10,3 \%)$ e hiperplasia $(10,9 \%)$. Na verdade, a depender da idade e da presença de fatores de risco, a incidência de câncer de endométrio relatada em pacientes que apresentam sangramento uterino na pós-menopausa varia na literatura pesquisada entre 1 e $25 \%$, sendo classicamente citada como de $10 \%{ }^{3,4}$.

Os trabalhos que avaliam o resultado histológico nas pacientes na pós-menopausa mostram freqüência de endométrio funcionante bastante variável 13,17,18. Nos poucos casos de endométrio funcionante encontrados em nossa pesquisa as pacientes tinham menos de cinco anos de menopausa, o que sugere a persistência de atividade estrogênica endógena. Dados semelhantes foram encontrados por Pontes et al. ${ }^{16}$.
Assim como outros autores ${ }^{13,16-18,20}$, verificamos percentual elevado de material insuficiente (16\%). Isto se deve, possivelmente, ao fato de que $48,1 \%$ das pacientes encontravam-se com mais de 60 anos de idade e 66,2\% delas tinham mais de cinco anos de menopausa, fatores estes que estão associados ao processo de atrofia endometrial. $\mathrm{O}$ material insuficiente traduziria, portanto, estado de intensa atrofia, dificultando ou mesmo impossibilitando a obtenção de material para estudo histopatológico.

Embora muitos estudos clássicos tenham usado a curetagem uterina como forma de coleta de material endometrial para análise histopatológica $^{13,18}$, sabemos que este método, assim como todos os outros que realizam coleta de tecido endometrial às cegas, possuem taxa de falso-negativos para diagnóstico de câncer em torno de $6 \%^{21-}$ ${ }^{23}$. Além disto, esse procedimento possui alto custo, requerendo hospitalização e anestesia para sua realização, cursando ainda com taxas mais elevadas de complicações ${ }^{14}$.

Em nosso estudo, todas as amostras endometriais foram obtidas durante o procedimento histeroscópico, método este que, de acordo com diversos estudos ${ }^{24,25}$, eleva bastante a acurácia diagnóstica. A grande vantagem é que a histeroscopia permite visão direta da cavidade uterina, diminuindo substancialmente falhas na identificação de lesões do endométrio, especialmente as focais ${ }^{19}$, além de orientar a biópsia. Na grande maioria dos casos este procedimento é realizado ambulatorialmente, sem necessidade de anestesia, o que, segundo alguns autores, reduz em cerca de $60 \%$ o número de internações decorrentes de sangramento uterino anormal na pós-menopausa, com queda substancial dos custos hospitalares $^{24-26}$.

Entre nós, Guimarães ${ }^{17}$ observou que os resultados histopatológicos corresponderam aos achados histeroscópicos em praticamente todos os casos, excetuando-se três ocasiões $(5,9 \%)$, nas quais se obteve material insuficiente, pela biópsia dirigida, para o diagnóstico histopatológico. No presente estudo, encontramos também boa concordância entre estes dois achados (kappa $=0,61)$, com o diagnóstico correto de 13 dos 16 casos de câncer. Ressalte-se que não foi encontrado nenhum caso de adenocarcinoma de endométrio dentre os endométrios atróficos diagnosticados histeroscopicamente.

Ainda em relação à histeroscopia, reconhece-se que, devido à falta de classificação uniforme das imagens histeroscópicas e à freqüente superposição destas em diferentes circunstâncias, seu uso isolado representa método limitado para o diagnóstico de lesões intra-uterinas ${ }^{4,11}$. Em 
conseqüência, recomenda-se que se deve sempre complementar este exame com a coleta de material para avaliação histopatológica. Como já foi ressaltado, em se procedendo desta maneira, a histeroscopia com biópsia de endométrio torna-se método de elevada acurácia diagnóstica, motivo pelo qual o utilizamos como padrão-ouro para o diagnóstico das lesões endometriais ${ }^{19,27}$.

Não obstante todas essas vantagens, a histeroscopia permanece ainda como método invasivo, requerendo profissionais treinados e aparelhagem de custo elevado para sua realização. Com o advento da ultra-sonografia transvaginal, permitindo maior resolução no estudo da cavidade endometrial, esta passou a ser adotada em diversos serviços para a propedêutica do sangramento uterino na pós-menopausa, surgindo em conseqüência diversos estudos para avaliar sua acurácia na detecção de lesões do endométrio nessa população ${ }^{27}$.

Em nosso estudo, efetuamos a comparação entre dois pontos de corte ( $4 \mathrm{~mm}$ e $5 \mathrm{~mm}$ ). Quando utilizamos $4 \mathrm{~mm}$ como ponto de corte, consideramos como tendo ultra-sonografia anormal 117 mulheres $(75 \%)$, das quais mais de $70 \%(n=86)$ tinham, na verdade, endométrio sem alterações histopatológicas. Entretanto, quando consideramos o ponto de corte de $5 \mathrm{~mm}$, não observamos modificação importante do percentual de resultados ecográficos alterados (em torno de 67\%) ( $p=0,7)$, sendo que a freqüência de exame histopatológico com lesões pré-malignas/malignas foi inferior a $30 \%$, verificando-se, portanto, percentual semelhante de falso-positivos.

Acreditamos, portanto, que o ponto de corte de $4 \mathrm{~mm}$ deve ser preferencialmente empregado porque, apesar do grande número de pacientes normais que terão de ser investigadas (baixa especificidade), esse número não difere significativamente quando se adota ponto de corte maior. Além disso, o ponto de corte de $4 \mathrm{~mm}$ é mais seguro, porque quando se adotou este limite não se observou nenhum caso de câncer de endométrio nas pacientes com eco endometrial normal.

Nosso estudo reforça, ainda, os resultados encontrados por Guimarães ${ }^{17}$, em estudo anterior realizado no IMIP, no que diz respeito à importância da ultra-sonografia para avaliar as pacientes com sangramento uterino na pós-menopausa. Apesar da indicação sistemática de investigação de todas as mulheres apresentando sangramento de origem uterina na pós-menopausa, uma vez que o câncer e suas lesões precursoras são encontrados em cerca de $20 \%$ dos casos, a ultra-sonografia pode ser utilizada como avaliação inicial destas pacientes ${ }^{17}$. Estamos de acordo com Karlsson et al. ${ }^{13}$ e Goldstein et $a l .{ }^{4}$ em utilizar 4 mm como ponto de corte para se excluir neoplasia endometrial, pois, como já ressaltamos, a taxa de falso-positivos é semelhante ao ponto de corte de $5 \mathrm{~mm}$ sem, contudo, apresentar nenhum resultado falso-negativo. É importante acrescentar que a introdução deste exame na propedêutica destas pacientes não apenas reduz os gastos com procedimentos hospitalares como também diminui a realização de procedimentos invasivos que, embora relativamente seguros, acarretam algumas complicações.

Diante do exposto, parece-nos seguro e prudente incentivar a avaliação das pacientes com sangramento uterino na pós-menopausa com a ultra-sonografia e prosseguir o seguimento apenas naquelas que ultrapassarem o limite de corte de $4 \mathrm{~mm}$ para medida da espessura do eco endometrial ou naquelas que possuam fatores de riscos importantes para o desenvolvimento do câncer de endométrio.

\section{ABSTRACT}

Purpose: to determine the association between ultrasonographic, hysteroscopic and histopathologic findings in women with postmenopausal uterine bleeding. Methods: a retrospective, cross-sectional study was conducted enrolling 156 women with postmenopausal bleeding attended at the Diagnostic Center - IMIP during the period of January 1995 to December 2001. According to the results of the endometrial examination (ultrasound), the patients were classified as having an abnormal or normal finding depending on the cutoffs of 4 or $5 \mathrm{~mm}$. The most common histologic and hysteroscopic findings were studied and classified as premalignant/malignant lesions (hyperplasial endometrial cancer) or benign findings. These results were compared using the kappa index, to establish the agreement between these techniques.

Results: the frequency of endometrial thickening was 75.0 and $67.3 \%$ according to cutoffs of $4 \mathrm{~mm}$ and $5 \mathrm{~mm}$, respectively. Hysteroscopic findings were atrophic endometrium in 59 (37.8\%), endometrial polyp in 56 (35.9\%), endometrial hyperplasia in $17(10.9 \%)$, cancer in 16 (10.3\%), still active endometrium in $1(3,2)$, and other findings in $3(1.9 \%)$. The two most frequent histopathologic findings were also atrophic endometrium (31.4\%) and endometrial polyps $(26, .3 \%)$, followed by scanty material $(16.0 \%)$, endometrial cancer (10.9\%), endometrial hyperplastic changes (9.0\%), and others (6.4\%). A good agreement between hysteroscopic and histopathologic findings was observed (kappa $=0.61$ ).

Conclusions: the frequency of endometrial thickening was 75.0 and $67.3 \%$ according to cutoffs of $4 \mathrm{~mm}$ and $5 \mathrm{~mm}$, respectively. No premalignant or malignant lesions were missed when an endometrial cutoff of $4 \mathrm{~mm}$ was used. The most frequent hysteroscopic and histopathologic findings 
were atrophic endometrium and endometrial polyps and a good agreement between these findings was encountered.

KEYWORDS: Postmenopausal bleeding. Endometrial thickness. Ultrasonography. Hysteroscopy. Endometrium: evaluation.

\section{Referências}

1. McKinlay SM. The normal menopause transition: an overview. Maturitas 1996; 23:137-45.

2. Speroff L. The perimenopause: definitions, demography, and physiology. Obstet Gynecol Clin North Am 2002; 29:397-410.

3. Rose PG. Endometrial carcinoma. N Engl J Med 1996; 335:640-9.

4. Goldstein RB, Bree RL, Benson CB, et al. Evaluation of the woman with postmenopausal bleeding: Society of Radiologists in Ultrasound-Sponsored Consensus Conference statement. J Ultrasound Med 2001; 20:1025-36.

5. Diczfalusy E. Menopause, developing countries and the $21^{\text {st }}$ century. Acta Obstet Gynecol Scand Suppl $1986 ; 134: 45-57$.

6. Utian WH. Overview on menopause. Am J Obstet Gynecol 1987; 156:1280-3.

7. Hamou J. Hysteroscopy and microhysteroscopy. $1^{\text {st }}$ ed. Norwalk: Appleton \& Lange; 1991.

8. Buyuk E, Durmusoglu F, Erenus M, Karakol B. Endometrial disease diagnosed by transvaginal ultrasound and dilatation and curettage. Acta Obstet Gynecol Scand 1999; 78:419-22.

9. Gull B, Karlsson B, Milsom I, Granberg S. Can ultrasound replace dilation and curettage? A longitudinal evaluation of postmenopausal bleeding and transvaginal sonographic measurement of the endometrium as predictors of endometrial cancer. Am J Obstet Gynecol 2003; 188:401-8.

10.Stock R, Kanbour A. Prehysterectomy curettage. Obstet Gynecol 1975; 45:537-41.

11.Loverro G, Bettocchi S, Cormio G, et al. Transvaginal sonography and hysteroscopy in posmenopausal uterine bleeding. Maturitas 1999; 33:139-44.

12.Giusa-Chiferi MG, Goncalves WJ, Baracat EC, de Albuquerque Neto LC, Bortoletto CC, de Lima GR. Transvaginal ultrasound, uterine biopsy and hysteroscopy for postmenopausal bleeding. Int $\mathrm{J}$ Gynaecol Obstet 1996; 55:39-44.

13. Karlsson B, Granberg S, Wikland M, et al. Transvaginal ultrasonography of the endometrium in women with postmenopausal bleeding: a Nordic multicenter study. Am J Obstet Gynecol 1995; 172:1488-94.
14.Grimes D. Diagnostic dilatation and curettage: a reappraisal. Am J Obstet Gynecol 1982; 142:1-6.

15. Mencaglia L. Hysteroscopy and adenocarcinoma. Obstet Gynecol Clin North Am 1995; 22:573-9.

16.Pontes A, Franco M, Nahas EAP, et al. Sangramento na pós-menopausa: avaliação clínica e histopatólogica. Reprod Clim 2000; 15:100-5.

17.Guimarães A. Acurácia da medida da espessura endometrial pela ultra-sonografia transvaginal, na detecção de lesões do endométrio em mulheres com sangramento uterino na pós-menopausa [dissertação]. Recife: Inst. Materno Infantil de Pernambuco; 1998.

18.Gredmark T, Kvint S, Havel G, Mattsson L. Histopathological findings in women with posmenopausal bleeding. Br J Obstet Gynaecol 1995; 102:133-6.

19.Epstein E, Ramirez A, Skoog L, Valentin L. Dilatation and curettage fails to detect most focal lesions in the uterine cavity in women with postmenopausal bleeding. Acta Obstet Gynecol Scand 2001; 80:1131-6.

20.Garcia MGM, Carvalho MGF, Garcia MM. Análise de fatores de risco em pacientes com adenocarcinoma endometrial. Reprod Clim 1998; 13:232-6.

21.Van den Bosch T, Vandendael A, Van Schoubroeck D, Wranz PA, Lombard CJ. Combining vaginal ultrasonography and office endometrial sampling in the diagnosis of endometrial disease in postmenopausal women. Obstet Gynecol 1995; 85:349-52.

22.Stovall TG, Solomon SK, Ling FW. Endometrial sampling prior to hysterectomy. Obstet Gynecol 1989; 73:405-9.

23. Gupta JK, Chien PF, Voit D, Clark TJ, Khan KS. Ultrasonographic endometrial thickness for diagnosing endometrial pathology in women with postmenopausal bleeding: a meta-analysis. Acta Obstet Gynecol Scand 2002; 81:799-816.

24. Sousa R, Silvestre M, Almeida e Sousa L, et al. Transvaginal ultrasonography and hysteroscopy in postmenopusal bleeding: a prospective study. Acta Obstet Gynecol Scand 2000; 80:856-62.

25.Garuti G, Sambruni I, Cellani F, Garzia D, Alleva P, Luerti M. Hysteroscopy and transvaginal ultrasonography in postmenopausal women with uterine bleeding. Int J Gynaecol Obstet 1999; 65:2533.

26.Fleischer AC, Kalemeris GC, Machin JE, Entman SS, James AE Jr. Sonographic depiction of normal and abnormal endometrium with histopathologic correlation. J Ultrasound Med 1986; 5:445-52.

27.Goldstein SR, Nachtigall M, Snyder JR, Nachtigall L. Endometrial assessment by vaginal ultrasonography before endometrial sampling in patients with postmenopausal bleeding. Am J Obstet Gynecol 1990; 163:119-23. 


\section{Laboratório: Bristol Anúncio: Tequin}

